\title{
Fuzzy approximation of Euler-Lagrange quadratic mappings
}

\author{
Hark-Mahn Kim¹, John M Rassias² and Juri Lee ${ }^{1 *}$
}

"Correspondence:
annans@hanmail.net
'Department of Mathematics,
Chungnam National University,
Daejeon, 305-764, Korea
Full list of author information is
available at the end of the article

available at the end of the article

\begin{abstract}
In this article, we consider the Hyers-Ulam stability of the Euler-Lagrange quadratic functional equation$$
f(k x+l y)+f(k x-l y)=k l[f(x+y)+f(x-y)]+2(k-l)[k f(x)-\mid f(y)]
$$

in fuzzy Banach spaces, where $k$, I are nonzero rational numbers with $k \neq 1$.
\end{abstract}

\section{Introduction}

The theory of fuzzy spaces has much progressed as the theory of randomness has developed. Some mathematicians have defined fuzzy norms on a vector space from various points of view [1-5]. Following Cheng and Mordeson [6], Bag and Samanta [1] gave the idea of fuzzy norm in such a manner that the corresponding fuzzy metric is of Kramosil and Michalek type [7] and investigated some properties of fuzzy normed spaces [8].

We use the definition of fuzzy normed spaces given in $[1,4,9]$.

Definition $1.1[1,4,9]$ Let $X$ be a real vector space. A function $N: X \times \mathbf{R} \rightarrow[0,1]$ is said to be a fuzzy norm on $X$ if for all $x, y \in X$ and all $s, t \in \mathbf{R}$,

$\left(\mathrm{N}_{1}\right) \quad N(x, t)=0$ for $t \leq 0$

$\left(\mathrm{N}_{2}\right) \quad x=0$ if and only if $N(x, t)=1$ for all $t>0$;

$\left(\mathrm{N}_{3}\right) N(c x, t)=N\left(x, \frac{t}{|c|}\right)$ for $c \neq 0$;

$\left(\mathrm{N}_{4}\right) N(x+y, s+t) \geq \min \{N(x, s), N(y, t)\}$;

$\left(\mathrm{N}_{5}\right) N(x, \cdot)$ is a non-decreasing function on $\mathbf{R}$ and $\lim _{t \rightarrow \infty} N(x, t)=1$;

$\left(\mathrm{N}_{6}\right)$ for $x \neq 0, N(x, \cdot)$ is continuous on $\mathbf{R}$.

The pair $(X, N)$ is called a fuzzy normed vector space. The properties of fuzzy normed vector spaces and examples of fuzzy norms are given in $[4,10]$.

Definition 1.2 $[1,4,9]$ Let $(X, N)$ be a fuzzy normed vector space. A sequence $\left\{x_{n}\right\}$ in $X$ is said to be convergent or to converges to $x$ if there exists an $x \in X$ such that $\lim _{n \rightarrow \infty} N\left(x_{n}-\right.$ $x, t)=1$ for all $t>0$. In this case, $x$ is called the limit of the sequence $\left\{x_{n}\right\}$, and we denote it by $N-\lim _{n \rightarrow \infty} x_{n}=x$.

@ 2013 Kim et al.; licensee Springer. This is an Open Access article distributed under the terms of the Creative Commons Attribution License (http://creativecommons.org/licenses/by/2.0), which permits unrestricted use, distribution, and reproduction in any medium, provided the original work is properly cited. 
Definition $1.3[1,4,9]$ Let $(X, N)$ be a fuzzy normed vector space. A sequence $\left\{x_{n}\right\}$ in $X$ is called Cauchy if for each $\varepsilon>0$ and each $t>0$, there exists an $n_{0} \in \mathbf{N}$ such that for all $n \geq n_{0}$ and all $p>0$, we have $N\left(x_{n+p}-x_{n}, t\right)>1-\varepsilon$.

It is well known that every convergent sequence in a fuzzy normed space is a Cauchy sequence. If each Cauchy sequence is convergent, then the fuzzy norm is said to be complete and the fuzzy normed vector space is called a fuzzy Banach space.

We say that a mapping $f: X \rightarrow Y$ between fuzzy normed spaces $X$ and $Y$ is continuous at $x_{0} \in X$ if for each sequence $\left\{x_{n}\right\}$ converging to each $x_{0} \in X$, the sequence $\left\{f\left(x_{n}\right)\right\}$ converges to $f\left(x_{0}\right)$. If $f: X \rightarrow Y$ is continuous at each $x \in X$, then $f: X \rightarrow Y$ is said to be continuous on $X$ (see [8]).

We recall the fixed point theorem from [11], which is needed in Section 4.

Let $X$ be a set. A function $d: X \times X \rightarrow[0, \infty]$ is called a generalized metric on $X$ if $d$ satisfies:

(1) $d(x, y)=0$ if and only if $x=y$;

(2) $d(x, y)=d(y, x)$ for all $x, y \in X$;

(3) $d(x, z) \leq d(x, y)+d(y, z)$ for all $x, y, z \in X$.

Theorem 1.4 $[11,12]$ Let $(X, d)$ be a complete generalized metric space and let $J: X \rightarrow X$ be a strictly contractive mapping with the Lipschitz constant $L<1$. Then, for each given element $x \in X$, either

$$
d\left(J^{n} x, J^{n+1} x\right)=\infty
$$

for all nonnegative integers $n$ or there exists a positive integer $n_{0}$ such that

(1) $d\left(J^{n} x, J^{n+1} x\right)<\infty, \forall n \geq n_{0}$;

(2) the sequence $\left\{J^{n} x\right\}$ converges to a fixed point $y^{*}$ of $J$;

(3) $y^{*}$ is the unique fixed point of $J$ in the set $Y=\left\{y \in X \mid d\left(J^{n_{0}} x, y\right)<\infty\right\}$;

(4) $d\left(y, y^{*}\right) \leq \frac{1}{1-L} d(y, J y)$ for all $y \in Y$.

In 1996, Isac and TM Rassias [13] were the first to provide a new application of fixed point theorems to prove the of stability theory of functional equations. By using fixed point methods, the stability problems of several functional equations have been extensively investigated by a number of authors (see [10-12, 14-17]).

The stability problem of functional equations originated from a question of Ulam [18] concerning the stability of group homomorphisms. Hyers [19] gave the first affirmative partial answer to the question of Ulam for additive mappings on Banach spaces. Hyers's theorem was generalized by Aoki [20] for additive mappings and by TM Rassias [21] for linear mappings by considering an unbounded Cauchy difference. A generalization of the TM Rassias theorem was obtain by Găvruta [22] by replacing the unbounded Cauchy difference by a general control function in the spirit of TM Rassias's approach.

The functional equation

$$
f(x+y)+f(x-y)=2 f(x)+2 f(y)
$$

is called a quadratic functional equation. In particular, every solution of the quadratic functional equation is said to be a quadratic function. A Hyers-Ulam stability problem for 
the quadratic functional equation was proved by Skof [23] for mappings $f: X \rightarrow Y$, where $X$ is a normed space and $Y$ is a Banach space. Cholewa [24] noticed that the theorem of Skof is still true if the relevant domain $X$ is replaced by an Abelian group. Czerwik [25] proved the Hyers-Ulam stability of the quadratic functional equation. In particular, JM Rassias investigated the Hyers-Ulam stability for the relative Euler-Lagrange functional equation

$$
f(a x+b y)+f(b x-a y)=\left(a^{2}+b^{2}\right)[f(x)+f(y)]
$$

in $[26,27]$. The stability problems of several functional equations have been extensively investigated by a number of authors, and there are many interesting results concerning this problem (see [28-32]).

In [33], Kim, Lee and Son have investigated the Hyers-Ulam stability of the quadratic functional equation

$$
f(k x+y)+f(k x-y)=k f(x+y)+k f(x-y)+2(k-1)[k f(x)-f(y)]
$$

for any fixed integer $k$ with $k \neq 0,1$.

In this paper, we prove the generalized Hyers-Ulam stability of the Euler-Lagrange quadratic functional equation

$$
f(k x+l y)+f(k x-l y)=k l[f(x+y)+f(x-y)]+2(k-l)[k f(x)-l f(y)]
$$

in fuzzy Banach spaces, where $k, l$ are nonzero rational numbers with $k \neq l$.

\section{General solution of (2)}

Lemma 2.1 A mapping $f: X \rightarrow Y$ between linear spaces satisfies the functional equation

$$
f(k x+y)+f(k x-y)=k f(x+y)+k f(x-y)+2(k-1)[k f(x)-f(y)]
$$

for any fixed rational number $k$ with $k \neq 0,1$ if and only if $f$ is quadratic.

Proof Let $f$ be a solution of equation (1). Letting $x=y=0$ in (1), we have $f(0)=0$. Putting $y=0$ in (1), we get $f(k x)=k^{2} f(x)$. Putting $x=0$ in (1), we get $f(-y)=f(y)$. Thus, the mapping $f$ is even. Therefore, it suffices to prove that if a mapping $f$ satisfies equation (1) for any fixed rational $k$ with $k \neq 0,1$, then $f$ is quadratic. Now, replacing $y$ by $x+y$ in (1), we have

$$
\begin{aligned}
& f((k+1) x+y)+f((k-1) x-y) \\
& \quad=k f(2 x+y)+k f(y)+2 k(k-1) f(x)-2(k-1) f(x+y)
\end{aligned}
$$

for all $x, y \in X$. Replacing $y$ by $-y$ in (3), we obtain

$$
\begin{aligned}
& f((k+1) x-y)+f((k-1) x+y) \\
& \quad=k f(2 x-y)+k f(y)+2 k(k-1) f(x)-2(k-1) f(x-y)
\end{aligned}
$$


for all $x, y \in X$. Adding (3) to (4), we get

$$
\begin{gathered}
f((k+1) x+y)+f((k+1) x-y)+f((k-1) x+y)+f((k-1) x-y) \\
\quad=k[f(2 x+y)+f(2 x-y)]-2(k-1)[f(x+y)+f(x-y)] \\
\quad=-2(k-1)[f(x+y)+f(x-y)]+4 k(k-1) f(x)+2 k f(y)
\end{gathered}
$$

for all $x, y \in X$. From the substitution $y:=k x+y$ in (1), we have

$$
\begin{array}{r}
f(2 k x+y)+f(y)=k[f((k+1) x+y)+f((k-1) x+y)] \\
+2 k(k-1) f(x)-2(k-1) f(k x+y)
\end{array}
$$

for all $x, y \in X$. Replacing $y$ by $-y$ in (6), we get

$$
\begin{aligned}
f(2 k x-y)+f(y)= & k[f((k+1) x-y)+f((k-1) x-y)] \\
& +2 k(k-1) f(x)-2(k-1) f(k x-y)
\end{aligned}
$$

for all $x, y \in X$. Adding (6) to (7), we get

$$
\begin{aligned}
f(2 k x+y)+f(2 k x-y)= & k[f((k+1) x+y)+f((k+1) x-y)] \\
& +k[f((k-1) x+y)+f((k-1) x-y)] \\
& -2(k-1)[f(k x+y)+f(k x-y)] \\
& +4 k(k-1) f(x)-2 f(y)
\end{aligned}
$$

for all $x, y \in X$. It follows from (8), by using (1) and (5), that

$$
\begin{aligned}
f(2 k x+y)+f(2 k x-y)= & k^{2}[f(2 x+y)+f(2 x-y)] \\
& -4 k(k-1)[f(x+y)+f(x-y)] \\
& +8 k(k-1) f(x)+2(k-1)(3 k-1) f(y)
\end{aligned}
$$

for all $x, y \in X$. If we replace $x$ by $2 x$ in (1), then we obtain that

$$
\begin{aligned}
f(2 k x+y)+f(2 k x-y)= & k[f(2 x+y)+f(2 x-y)] \\
& +2 k(k-1) f(2 x)-2(k-1) f(y)
\end{aligned}
$$

for all $x, y \in X$. Associating (9) with (10), we conclude that the mapping $f$ satisfies the equation

$$
f(2 x+y)+f(2 x-y)=4[f(x+y)+f(x-y)]+2[f(2 x)-4 f(x)]-6 f(y)
$$

for all $x, y \in X$. Then $f(x)=Q_{1}(x)+Q_{2}(x)$ for all $x \in X$, where $Q_{1}$ is quadratic and $Q_{2}$ is quartic by the papers [34-36]. Therefore, $f$ is quadratic because of the property $f(k x)=$ $k^{2} f(x)$.

Conversely, if a mapping $f$ is quadratic, then it is obvious that $f$ satisfies (1). 
Theorem 2.2 A mapping $f: X \rightarrow Y$ with $f(0)=0$ between linear spaces satisfies the functional equation (2) if and only iff is quadratic.

Proof Let $f$ be a solution of equation (2) and $f(0)=0$. Putting $y=0$ in (2), we get $f(k x)=$ $k^{2} f(x)$ for all $x \in X$. Putting $x=0$ and replacing $y$ by $x$, we have

$$
f(l x)+f(-l x)=l(2 l-k) f(x)+k l f(-x)
$$

for all $x \in X$. Replacing $x$ by $-x$ in (11), one gets

$$
f(-l x)+f(l x)=l(2 l-k) f(-x)+k l f(x)
$$

for all $x \in X$. Subtracting equation (11) from (12), we find $f(-x)=f(x)$ and so $f(l x)=l^{2} f(x)$ for all $x \in X$. Thus equation (2) can be rewritten as

$$
f(a x+y)+f(a x-y)=a f(x+y)+a f(x-y)+2 a(a-1) f(x)-2(a-1) f(y),
$$

where $a:=\frac{k}{l} \neq 0,1$ for all $x, y \in X$. Therefore, it follows from Lemma 2.1 that $f$ is quadratic. Conversely, if a mapping $f$ is quadratic, then it is obvious that $f$ satisfies equation (2).

\section{Stability of equation (2) by direct method}

Throughout this paper, we assume that $X$ is a linear space, $(Y, N)$ is a fuzzy Banach space and $\left(Z, N^{\prime}\right)$ is a fuzzy normed space.

For notational convenience, given a mapping $f: X \rightarrow Y$, we define the difference operator $D_{k l} f: X^{2} \rightarrow Y$ of equation (2) by

$$
D_{k l} f(x, y):=f(k x+l y)+f(k x-l y)-k l[f(x+y)+f(x-y)]-2(k-l)[k f(x)-l f(y)]
$$

for all $x, y \in X$.

Theorem 3.1 Assume that a mapping $f: X \rightarrow Y$ with $f(0)=0$ satisfies the inequality

$$
N\left(D_{k l} f(x, y), t\right) \geq N^{\prime}(\varphi(x, y), t)
$$

and $\varphi: X^{2} \rightarrow Z$ is a mapping for which there is a constant $s \in \mathbf{R}$ satisfying $0<|s|<k^{2}$ such that

$$
N^{\prime}(\varphi(k x, k y), t) \geq N^{\prime}(s \varphi(x, y), t)
$$

for all $x \in X$ and all $t>0$. Then we can find a unique quadratic mapping $Q: X \rightarrow Y$ satisfying the equation $D_{k l} Q(x, y)=0$ and the inequality

$$
N(f(x)-Q(x), t) \geq N^{\prime}\left(\frac{\varphi(x, 0)}{2\left(k^{2}-|s|\right)}, t\right), \quad t>0
$$

for all $x \in X$. 
Proof We observe from (14) that

$$
\begin{aligned}
& N^{\prime}\left(\varphi\left(k^{n} x, k^{n} y\right), t\right) \geq N^{\prime}\left(s^{n} \varphi(x, y), t\right)=N^{\prime}\left(\varphi(x, y), \frac{t}{|s|^{n}}\right), \quad t>0, \\
& N^{\prime}\left(\varphi\left(k^{n} x, k^{n} y\right),|s|^{n} t\right) \geq N^{\prime}(\varphi(x, y), t), \quad t>0
\end{aligned}
$$

for all $x, y \in X$. Putting $y:=0$ in (13), we obtain

$$
\begin{aligned}
& N\left(2 f(k x)-2 k^{2} f(x), t\right) \geq N^{\prime}(\varphi(x, 0), t), \quad \text { or } \\
& N\left(f(x)-\frac{f(k x)}{k^{2}}, \frac{t}{2 k^{2}}\right) \geq N^{\prime}(\varphi(x, 0), t)
\end{aligned}
$$

for all $x \in X$. Therefore it follows from (16), (17) that

$$
N\left(\frac{f\left(k^{n} x\right)}{k^{2 n}}-\frac{f\left(k^{n+1} x\right)}{k^{2(n+1)}}, \frac{|s|^{n} t}{2 k^{2(n+1)}}\right) \geq N^{\prime}\left(\varphi\left(k^{n} x, 0\right),|s|^{n} t\right) \geq N^{\prime}(\varphi(x, 0), t)
$$

for all $x \in X$ and any integer $n \geq 0$. So,

$$
\begin{aligned}
N\left(f(x)-\frac{f\left(k^{n} x\right)}{k^{2 n}}, \sum_{i=0}^{n-1} \frac{|s|^{i} t}{2 k^{2(i+1)}}\right) & =N\left(\sum_{i=0}^{n-1}\left(\frac{f\left(k^{i} x\right)}{k^{2 i}}-\frac{f\left(k^{i+1} x\right)}{k^{2(i+1)}}\right), \sum_{i=0}^{n-1} \frac{|s|^{i} t}{2 k^{2(i+1)}}\right) \\
& \geq \min _{0 \leq i \leq n-1}\left\{N\left(\frac{f\left(k^{i} x\right)}{k^{2 i}}-\frac{f\left(k^{i+1} x\right)}{k^{2(i+1)}}, \frac{|s|^{i} t}{2 k^{2(i+1)}}\right)\right\} \\
& \geq N^{\prime}(\varphi(x, 0), t), \quad t>0,
\end{aligned}
$$

which yields

$$
\begin{aligned}
& N\left(\frac{f\left(k^{m} x\right)}{k^{2 m}}-\frac{f\left(k^{m+p} x\right)}{k^{2(m+p)}}, \sum_{i=m}^{m+p-1} \frac{|s|^{i} t}{2 k^{2(i+1)}}\right) \\
& \quad=N\left(\sum_{i=m}^{m+p-1}\left(\frac{f\left(k^{i} x\right)}{k^{2 i}}-\frac{f\left(k^{i+1} x\right)}{k^{2(i+1)}}\right), \sum_{i=m}^{m+p-1} \frac{|s|^{i} t}{2 k^{2(i+1)}}\right) \\
& \geq \min _{m \leq i \leq m+p-1}\left\{N\left(\frac{f\left(k^{i} x\right)}{k^{2 i}}-\frac{f\left(k^{i+1} x\right)}{k^{2(n+1)}}, \frac{|s|^{i} t}{2 k^{2(i+1)}}\right)\right\} \\
& \geq N^{\prime}(\varphi(x, 0), t), \quad t>0,
\end{aligned}
$$

for all $x \in X$ and any integers $p>0, m \geq 0$. Hence, one obtains

$$
N\left(\frac{f\left(k^{m} x\right)}{k^{2 m}}-\frac{f\left(k^{m+p} x\right)}{k^{2(m+p)}}, t\right) \geq N^{\prime}\left(\varphi(x, 0), \frac{t}{\sum_{i=m}^{m+p-1} \frac{|s|^{i}}{2 k^{2(i+1)}}}\right)
$$

for all $x \in X$ and any integers $p>0, m \geq 0, t>0$. Since $\sum_{i=m}^{m+p-1} \frac{|s|^{i}}{k^{2 i}}$ is a convergent series, we see, by taking the limit $m \rightarrow \infty$ in the last inequality, that the sequence $\left\{\frac{f\left(k^{n} x\right)}{k^{2 n}}\right\}$ is Cauchy in the fuzzy Banach space $(Y, N)$ and so it converges in $Y$. Therefore a mapping $Q: X \rightarrow Y$ defined by

$$
Q(x):=N-\lim _{n \rightarrow \infty} \frac{f\left(k^{n} x\right)}{k^{2 n}}
$$


is well defined for all $x \in X$. It means that $\lim _{n \rightarrow \infty} N\left(\frac{f\left(k^{n} x\right)}{k^{2 n}}-Q(x), t\right)=1, t>0$, for all $x \in X$. In addition, we see from (18) that

$$
N\left(f(x)-\frac{f\left(k^{n} x\right)}{k^{2 n}}, t\right) \geq N^{\prime}\left(\varphi(x, 0), \frac{t}{\sum_{i=0}^{n-1} \frac{|s|^{i}}{2 k^{2(i+1)}}}\right)
$$

and so

$$
\begin{aligned}
N(f(x)-Q(x), t) & \geq \min \left\{N\left(f(x)-\frac{f\left(k^{n} x\right)}{k^{2 n}},(1-\varepsilon) t\right), N\left(\frac{f\left(k^{n} x\right)}{k^{2 n}}-Q(x), \varepsilon t\right)\right\} \\
& \geq N^{\prime}\left(\varphi(x, 0), \frac{(1-\varepsilon) t}{\sum_{i=0}^{n-1} \frac{|s|^{i}}{2 k^{2(i+1)}}}\right) \\
& \geq N^{\prime}\left(\varphi(x, 0), 2(1-\varepsilon)\left(k^{2}-|s|\right) t\right), \quad 0<\varepsilon<1,
\end{aligned}
$$

for sufficiently large $n$ and for all $x \in X$ and all $t>0$. Since $\varepsilon$ is arbitrary and $N^{\prime}$ is left continuous, we obtain

$$
N(f(x)-Q(x), t) \geq N^{\prime}\left(\varphi(x, 0), 2\left(k^{2}-|s|\right) t\right), \quad t>0,
$$

for all $x \in X$, which yields the approximation (15).

In addition, it is clear from (13) and $\left(\mathrm{N}_{5}\right)$ that the relation

$$
\begin{aligned}
N\left(\frac{D_{k l} f\left(k^{n} x, k^{n} y\right)}{k^{2 n}}, t\right) & \geq N^{\prime}\left(\varphi\left(k^{n} x, k^{n} y\right), k^{2 n} t\right) \\
& \geq N^{\prime}\left(\varphi(x, y), \frac{k^{2 n}}{|s|^{n}} t\right) \\
& \rightarrow 1 \quad \text { as } n \rightarrow \infty
\end{aligned}
$$

holds for all $x, y \in X$ and all $t>0$. Therefore, we obtain by use of $\lim _{n \rightarrow \infty} N\left(\frac{f\left(k^{n} x\right)}{k^{2 n}}-Q(x), t\right)=$ $1(t>0)$ that

$$
\begin{aligned}
N & \left(D_{k l} Q(x, y), t\right) \\
& \geq \min \left\{N\left(D_{k l} Q(x, y)-\frac{D_{k l} f\left(k^{n} x, k^{n} y\right)}{k^{2 n}}, \frac{t}{2}\right), N\left(\frac{D_{k l} f\left(k^{n} x, k^{n} y\right)}{k^{2 n}}, \frac{t}{2}\right)\right\} \\
& \left.=N\left(\frac{D_{k l} f\left(k^{n} x, k^{n} y\right)}{k^{2 n}}, \frac{t}{2}\right) \quad \text { (for sufficiently large } n\right) \\
& \geq N^{\prime}\left(\varphi(x, y), \frac{k^{2 n}}{2|s|^{n}} t\right), \quad t>0 \\
& \rightarrow 1 \quad \text { as } n \rightarrow \infty,
\end{aligned}
$$

which implies $D_{k l} Q(x, y)=0$ by $\left(\mathrm{N}_{2}\right)$. Thus we find that $Q$ is a quadratic mapping satisfying equation (2) and inequality (15) near the approximate quadratic mapping $f: X \rightarrow Y$.

To prove the aforementioned uniqueness, we assume now that there is another quadratic mapping $Q^{\prime}: X \rightarrow Y$ which satisfies inequality (15). Then one establishes by the equality 


$$
\begin{aligned}
& Q^{\prime}\left(k^{n} x\right)=k^{2 n} Q(x) \text { and }(15) \text { that } \\
& \begin{aligned}
N\left(Q(x)-Q^{\prime}(x), t\right) & =N\left(\frac{Q\left(k^{n} x\right)}{k^{2 n}}-\frac{Q^{\prime}\left(k^{n} x\right)}{k^{2 n}}, t\right) \\
& \geq \min \left\{N\left(\frac{Q\left(k^{n} x\right)}{k^{2 n}}-\frac{f\left(k^{n} x\right)}{k^{2 n}}, \frac{t}{2}\right), N\left(\frac{f\left(k^{n} x\right)}{k^{2 n}}-\frac{Q^{\prime}\left(k^{n} x\right)}{k^{2 n}}, \frac{t}{2}\right)\right\} \\
& \geq N^{\prime}\left(\varphi\left(k^{n} x, 0\right),\left(k^{2}-|s|\right) k^{2 n} t\right) \\
& \geq N^{\prime}\left(\varphi(x, 0), \frac{\left(k^{2}-|s|\right) k^{2 n} t}{|s|^{n}}\right), \quad t>0,
\end{aligned}
\end{aligned}
$$

for all $n \in \mathbf{N}$, which tends to 1 as $n \rightarrow \infty$ by $\left(\mathrm{N}_{5}\right)$. Therefore one obtains $Q(x)=Q^{\prime}(x)$ for all $x \in X$, completing the proof of uniqueness.

We remark that if $k=1$ in Theorem 3.1, then $N^{\prime}(\varphi(x, y), t) \geq N^{\prime}\left(\varphi(x, y), \frac{t}{|s|^{n}}\right) \rightarrow 1$ as $n \rightarrow$ $\infty$, and so $\varphi(x, y)=0$ for all $x, y \in X$. Hence $D_{k l} f(x, y)=0$ for all $x, y \in X$ and $f$ is itself a quadratic mapping.

Theorem 3.2 Assume that a mapping $f: X \rightarrow Y$ with $f(0)=0$ satisfies the inequality

$$
N\left(D_{k l} f(x, y), t\right) \geq N^{\prime}(\varphi(x, y), t)
$$

and that $\varphi: X^{2} \rightarrow Z$ is a mapping for which there is a constant $s \in \mathbf{R}$ satisfying $|s|>k^{2}$ such that

$$
N^{\prime}\left(\varphi\left(\frac{x}{k}, \frac{y}{k}\right), t\right) \geq N^{\prime}\left(\frac{1}{s} \varphi(x, y), t\right), \quad t>0
$$

for all $x \in X$ and all $t>0$. Then we can find a unique quadratic mapping $Q: X \rightarrow Y$ satisfying the equation $D_{k l} Q(x, y)=0$ and the inequality

$$
N(f(x)-Q(x), t) \geq N^{\prime}\left(\frac{\varphi(x, 0)}{2\left(|s|-k^{2}\right)}, t\right), \quad t>0,
$$

for all $x \in X$.

Proof It follows from (17) and (23) that

$$
N\left(f(x)-k^{2} f\left(\frac{x}{k}\right), \frac{t}{2|s|}\right) \geq N^{\prime}(\varphi(x, 0), t), \quad t>0,
$$

for all $x \in X$. Therefore it follows that

$$
N\left(f(x)-k^{2 n} f\left(\frac{x}{k^{n}}\right), \sum_{i=0}^{n-1} \frac{k^{2 i}}{2|s|^{i+1}} t\right) \geq N^{\prime}(\varphi(x, 0), t), \quad t>0,
$$

for all $x \in X$ and any integer $n>0$. Thus we see from the last inequality that

$$
N\left(f(x)-k^{2 n} f\left(\frac{x}{k^{n}}\right), t\right) \geq N^{\prime}\left(\varphi(x, 0), \frac{t}{\sum_{i=0}^{n-1} \frac{k^{2 i}}{2|s|^{i+1}}}\right) \geq N^{\prime}\left(\varphi(x, 0), 2\left(|s|-k^{2}\right) t\right), \quad t>0 .
$$


The remaining assertion goes in a similar way as the corresponding part of Theorem 3.1.

We also observe that if $k=1$ in Theorem 3.2, then $N^{\prime}(\varphi(x, y), t) \geq N^{\prime}\left(\varphi(x, y),|s|^{n} t\right) \rightarrow 1$ as $n \rightarrow \infty$, and so $\varphi(x, y)=0$ for all $x, y \in X$. Hence, $D_{k l} f=0$ and $f$ is itself a quadratic mapping.

Corollary 3.3 Let $X$ be a normed space and $\left(\mathbf{R}, N^{\prime}\right)$ be a fuzzy normed space. Assume that there exist real numbers $\theta_{1} \geq 0, \theta_{2} \geq 0$ and that $p$ is a real number such that either $0<p<2$ or $p>2$. If a mapping $f: X \rightarrow Y$ with $f(0)=0$ satisfies the inequality

$$
N\left(D_{k l} f(x, y), t\right) \geq N^{\prime}\left(\theta_{1}\|x\|^{p}+\theta_{2}\|y\|^{p}, t\right)
$$

for all $x, y \in X$ and all $t>0$, then we can find a unique quadratic mapping $Q: X \rightarrow Y$ satisfying the equation $D_{k l} Q(x, y)=0$ and the inequality

$$
N(f(x)-Q(x), t) \geq \begin{cases}N^{\prime}\left(\frac{\theta_{1}\|x\|^{p}}{2\left(k^{2}-|k|^{p}\right)}, t\right), & \text { if } 0<p<2,|k|>1(p>2,|k|<1), \\ N^{\prime}\left(\frac{\theta_{1}\|x\|^{p}}{2\left(|k|^{p}-k^{2}\right)}, t\right), & \text { if } p>2,|k|>1(0<p<2,|k|<1)\end{cases}
$$

for all $x \in X$ and all $t>0$.

Proof Taking $\varphi(x, y)=\theta_{1}\|x\|^{p}+\theta_{2}\|y\|^{p}$ and applying Theorems 3.1 and 3.2, we obtain the desired approximation, respectively.

The following is a simple example that the quadratic functional equation $D_{k l} f(x, y)=0$, $k \geq 2, k>l \geq 1$ is not stable for $p=2$ in Corollary 3.3. This is a counterexample for the singular case $p=2$ in a real space with a fuzzy norm $N(x, t)=N^{\prime}(x, t)=\frac{t}{t+\|x\|}$.

Example 3.4 Let $\phi: \mathbf{R} \rightarrow \mathbf{R}$ be defined by

$$
\phi(x)= \begin{cases}\mu x^{2} & \text { if }|x|<1 \\ \mu & \text { otherwise }\end{cases}
$$

where $\mu>0$ is a positive constant, and define $f: \mathbf{R} \rightarrow \mathbf{R}$ by

$$
f(x)=\sum_{i=0}^{\infty} \frac{\phi\left(k^{i} x\right)}{k^{2 i}} \quad \text { for all } x \in \mathbf{R}
$$

Then $f$ satisfies the functional inequality

$$
\begin{aligned}
& |f(k x+l y)+f(k x-l y)-k l[f(x+y)+f(x-y)]-2 k(k-l)[k f(x)-l f(y)]| \\
& \quad \leq \frac{2 k^{6} \mu\left(k^{2}+k l-l^{2}+1\right)}{k^{2}-1}\left(|x|^{2}+|y|^{2}\right)
\end{aligned}
$$

for all $x, y \in \mathbf{R}$, but there do not exist a quadratic function $Q: \mathbf{R} \rightarrow \mathbf{R}$ and a constant $\beta>0$ such that

$$
|f(x)-Q(x)| \leq \beta|x|^{2} \quad \text { for all } x \in \mathbf{R} \text {. }
$$


Proof It is easy to see that $\phi$ is bounded by $\mu$ and $f$ is bounded by $\frac{k^{2} \mu}{k^{2}-1}$ on $\mathbf{R}$. First, if $|x|^{2}+|y|^{2} \geq \frac{1}{k^{4}}$ or 0 , then

$$
\left|D_{k l} f(x, y)\right| \leq \frac{k^{2} \mu}{k^{2}-1}\left\{2\left(k^{2}+k l-l^{2}+1\right)\right\} \leq \frac{2 k^{6} \mu\left(k^{2}+k l-l^{2}+1\right)}{k^{2}-1}\left(|x|^{2}+|y|^{2}\right)
$$

and thus (25) is true. Now suppose that $0<|x|^{2}+|y|^{2}<\frac{1}{k^{4}}$. Then there exists a positive integer $i_{0}$ such that

$$
\frac{1}{k^{2 i_{0}+3}} \leq|x|^{2}+|y|^{2}<\frac{1}{k^{2 i_{0}+2}}
$$

so that $k^{2 i_{0}}|x|^{2}<\frac{1}{k^{2}}, k^{2 i_{0}}|y|^{2}<\frac{1}{k^{2}}$ and $k^{i_{0}-1}(k x \pm l y), k^{i_{0}-1}(x \pm y), k^{i_{0}-1} x, k^{i_{0}-1} y$ all belong to the interval $(-1,1)$. Hence, for $i=0,1, \ldots, i_{0}-1$,

$$
D_{k l} \phi\left(k^{i} x, k^{i} y\right)=0
$$

since each term of $D_{k l} \phi\left(k^{i} x, k^{i} y\right)$ is defined by $\mu x^{2}$. Therefore, it follows from the definition of $f$ and inequality (27) that

$$
\begin{aligned}
\left|D_{k l} f(x, y)\right| & \leq \sum_{i=0}^{\infty} \frac{1}{k^{2 i}}\left|D_{k l} \phi\left(k^{i} x, k^{i} y\right)\right| \leq \sum_{i=i_{0}}^{\infty} \frac{1}{k^{2 i}}\left|D_{k l} \phi\left(k^{i} x, k^{i} y\right)\right| \\
& \leq \sum_{i=i_{0}}^{\infty} \frac{1}{k^{2 i}}\left\{2\left(k^{2}+k l-l^{2}+1\right)\right\} \mu=\frac{2 k^{2} \mu\left(k^{2}+k l-l^{2}+1\right)}{k^{2 i_{0}}\left(k^{2}-1\right)} \\
& \leq \frac{2 k^{5} \mu\left(k^{2}+k l-l^{2}+1\right)}{k^{2}-1}\left(|x|^{2}+|y|^{2}\right)
\end{aligned}
$$

for all $x, y \in \mathbf{R}$ with $0<|x|^{2}+|y|^{2}<\frac{1}{k^{4}}$. Thus $f$ satisfies inequality (25) for all $x, y \in \mathbf{R}$.

We claim that the quadratic functional equation $D_{k l} f(x, y)=0$ is not stable for $p=2$ in Corollary 3.3. Suppose on the contrary that there exist a quadratic mapping $Q: \mathbf{R} \rightarrow \mathbf{R}$ and a constant $\beta>0$ satisfying (26). Since $f$ is bounded and continuous for all $x \in \mathbf{R}$, $Q$ is bounded on any open interval containing the origin and continuous at the origin. Therefore, $Q$ must have the form $Q(x)=\eta x^{2}$ for any $x$ in $\mathbf{R}$. Thus we obtain that

$$
|f(x)| \leq(\beta+|\eta|)|x|^{2} \quad \text { for all } x \in \mathbf{R} \text {. }
$$

However, we can choose a positive integer $p$ with $p \mu>\beta+|\eta|$. Then if $x \in\left(0, \frac{1}{k^{p-1}}\right)$, then $k^{i} x \in(0,1)$ for all $i=0,1, \ldots, p-1$, and so for this $x$ we get

$$
f(x)=\sum_{i=0}^{\infty} \frac{\phi\left(k^{i} x\right)}{k^{2 i}} \geq \sum_{i=0}^{p-1} \frac{\mu\left(k^{i} x\right)^{2}}{k^{2 i}}=p \mu x^{2}>(\beta+|\eta|) x^{2}
$$

which contradicts (29). Therefore the quadratic functional equation $D_{k l} f(x, y)=0$ is not stable if $p=2=q$ is assumed in Corollary 3.3. 
Corollary 3.5 Assume that for $k \neq 1$, there exists a real number $\theta \geq 0$ such that the mapping $f: X \rightarrow Y$ with $f(0)=0$ satisfies the inequality

$$
N\left(D_{k l} f(x, y), t\right) \geq N^{\prime}(\theta, t)
$$

for all $x, y \in X$ and all $t>0$. Then we can find a unique quadratic mapping $Q: X \rightarrow Y$ satisfying the equation $D_{k l} Q(x, y)=0$ and the inequality

$$
N(f(x)-Q(x), t) \geq N^{\prime}\left(\frac{\theta}{2\left|k^{2}-1\right|}, t\right)
$$

for all $x \in X$ and all $t>0$.

We remark that if $\theta=0$, then $N\left(D_{k l} f(x, y), t\right) \geq N^{\prime}(0, t)=1$, and so $D_{k l} f(x, y)=0$. Thus we get $f=Q$ is itself a quadratic mapping.

\section{Stability of equation (2) by fixed point method}

Now, in the next theorem, we are going to consider a stability problem concerning the stability of equation (2) by using a fixed point theorem of the alternative for contraction mappings on generalized complete metric spaces due to Margolis and Diaz [11].

Theorem 4.1 Assume that there exists a constant $s \in \mathbf{R}$ with $|s| \neq 1$ and $q>0$ satisfying $0<|s|^{\frac{1}{q}}<k^{2}$ such that a mapping $f: X \rightarrow Y$ with $f(0)=0$ satisfies the inequality

$$
N\left(D_{k l} f(x, y), t_{1}+t_{2}\right) \geq \min \left\{N^{\prime}\left(\varphi(x), t_{1}^{q}\right), N^{\prime}\left(\varphi(y), t_{2}^{q}\right)\right\}
$$

for all $x, y \in X, t_{i}>0(i=1,2)$ and that $\varphi: X \rightarrow Z$ is a mapping satisfying

$$
N^{\prime}(\varphi(k x), t) \geq N^{\prime}(s \varphi(x), t)
$$

for all $x \in X$ and all $t>0$. Then there exists a unique quadratic mapping $Q: X \rightarrow Y$ satisfying the equation $D_{k l} Q(x, y)=0$ and the inequality

$$
N(f(x)-Q(x), t) \geq \min \left\{N^{\prime}\left(\frac{\varphi(x)}{\left(k^{2}-|s|^{\frac{1}{q}}\right)^{q}}, t^{q}\right), N^{\prime}\left(\frac{\varphi(0)}{\left(k^{2}-|s|^{\frac{1}{q}}\right)^{q}}, t^{q}\right)\right\}
$$

for all $x \in X$ and all $t>0$.

Proof We consider the set of functions

$$
\Omega:=\{g: X \rightarrow Y \mid g(0)=0\}
$$

and define a generalized metric on $\Omega$ as follows:

$$
\begin{aligned}
d_{\Omega}(g, h):= & \inf \left\{K \in[0, \infty]: N(g(x)-h(x), K t) \geq \min \left\{N^{\prime}\left(\varphi(x), t^{q}\right), N^{\prime}\left(\varphi(0), t^{q}\right)\right\},\right. \\
& \forall x \in X, \forall t>0\} .
\end{aligned}
$$

Then one can easily see that $\left(\Omega, d_{\Omega}\right)$ is a complete generalized metric space $[37,38]$. 
Now, we define an operator $J: \Omega \rightarrow \Omega$ as

$$
J g(x)=\frac{g(k x)}{k^{2}}
$$

for all $g \in \Omega, x \in X$.

We first prove that $J$ is strictly contractive on $\Omega$. For any $g, h \in \Omega$, let $\varepsilon \in[0, \infty)$ be any constant with $d_{\Omega}(g, h) \leq \varepsilon$. Then we deduce from the use of (31) and the definition of $d_{\Omega}(g, h)$ that

$$
\begin{aligned}
& N(g(x)-h(x), \varepsilon t) \geq \min \left\{N^{\prime}\left(\varphi(x), t^{q}\right), N^{\prime}\left(\varphi(0), t^{q}\right)\right\}, \quad \forall x \in X, t>0 \\
& \quad \Rightarrow \quad N\left(\frac{g(k x)}{k^{2}}-\frac{h(k x)}{k^{2}}, \frac{|s|^{\frac{1}{q}} \varepsilon t}{k^{2}}\right) \geq \min \left\{N^{\prime}\left(\varphi(k x),|s| t^{q}\right), N^{\prime}\left(\varphi(0),|s| t^{q}\right)\right\} \\
& \Rightarrow \quad N\left(J g(x)-J h(x), \frac{|s|^{\frac{1}{q}} \varepsilon t}{k^{2}}\right) \geq \min \left\{N^{\prime}\left(\varphi(x), t^{q}\right), N^{\prime}\left(\varphi(0), t^{q}\right)\right\}, \quad \forall x \in X, t>0 \\
& \Rightarrow \quad d_{\Omega}(J g, J h) \leq \frac{|s|^{\frac{1}{q}} \varepsilon}{k^{2}} .
\end{aligned}
$$

Since $\varepsilon$ is an arbitrary constant with $d_{\Omega}(g, h) \leq \varepsilon$, we see that for any $g, h \in \Omega$,

$$
d_{\Omega}(J g, J h) \leq \frac{|s|^{\frac{1}{q}}}{k^{2}} d_{\Omega}(g, h)
$$

which implies $J$ is strictly contractive with the constant $\frac{|s|^{\frac{1}{q}}}{k^{2}}<1$ on $\Omega$.

We now want to show that $d(f, J f)<\infty$. If we put $y:=0, t_{i}:=t(i=1,2)$ in (30), then we arrive at

$$
N\left(f(x)-\frac{f(k x)}{k^{2}}, \frac{t}{k^{2}}\right) \geq \min \left\{N^{\prime}\left(\varphi(x), t^{q}\right), N^{\prime}\left(\varphi(0), t^{q}\right)\right\}
$$

which yields $d_{\Omega}(f, J f) \leq \frac{1}{k^{2}}$ and so $d_{\Omega}\left(J^{n} f, J^{n+1} f\right) \leq d_{\Omega}(f, J f) \leq \frac{1}{k^{2}}$ for all $n \in \mathbf{N}$.

Using the fixed point theorem of the alternative for contractions on generalized complete metric spaces due to Margolis and Diaz [11], we see the following (i), (ii) and (iii):

(i) There is a mapping $Q: X \rightarrow Y$ with $Q(0)=0$ such that

$$
d_{\Omega}(f, Q) \leq \frac{1}{1-\frac{|s|^{\frac{1}{q}}}{k^{2}}} d_{\Omega}(f, J f) \leq \frac{1}{k^{2}-|s|^{\frac{1}{q}}}
$$

and $Q$ is a fixed point of the operator $J$, that is, $\frac{1}{k^{2}} Q(k x)=J Q(x)=Q(x)$ for all $x \in X$. Thus we can get

$$
\begin{aligned}
& N\left(f(x)-Q(x), \frac{t}{k^{2}-|s|^{\frac{1}{q}}}\right) \geq \min \left\{N^{\prime}\left(\varphi(x), t^{q}\right), N^{\prime}\left(\varphi(0), t^{q}\right)\right\} \\
& N(f(x)-Q(x), t) \geq \min \left\{N^{\prime}\left(\varphi(x),\left(k^{2}-|s|^{\frac{1}{q}}\right)^{q} t^{q}\right), N^{\prime}\left(\varphi(0),\left(k^{2}-|s|^{\frac{1}{q}}\right)^{q} t^{q}\right)\right\}
\end{aligned}
$$

for all $t>0$ and all $x \in X$. 
(ii) $d_{\Omega}\left(J^{n} f, Q\right) \rightarrow 0$ as $n \rightarrow \infty$. Thus we obtain

$$
\begin{aligned}
& N\left(\frac{f\left(k^{n} x\right)}{k^{2 n}}-Q(x), t\right) \\
& \quad=N\left(f\left(k^{n} x\right)-Q\left(k^{n} x\right), k^{2 n} t\right) \\
& \quad \geq \min \left\{N^{\prime}\left(\frac{\varphi\left(k^{n} x\right)}{\left(k^{2}-|s|^{\frac{1}{q}}\right)^{q}}, k^{2 n q} t^{q}\right), N^{\prime}\left(\frac{\varphi(0)}{\left(k^{2}-|s|^{\frac{1}{q}}\right)^{q}}, k^{2 n q} t^{q}\right)\right\} \\
& \quad=\min \left\{N^{\prime}\left(\frac{\varphi(x)}{\left(k^{2}-|s|^{\frac{1}{q}}\right)^{q}},\left(\frac{k^{2 q}}{|s|}\right)^{n} t^{q}\right), N^{\prime}\left(\frac{\varphi(0)}{\left(k^{2}-|s|^{\frac{1}{q}}\right)^{q}},\left(\frac{k^{2 q}}{|s|}\right)^{n} t^{q}\right)\right\} \\
& \quad \rightarrow 1 \quad \text { as } n \rightarrow \infty\left(\frac{k^{2 q}}{|s|}>1\right)
\end{aligned}
$$

for all $t>0$ and all $x \in X$, that is, the mapping $Q: X \rightarrow Y$ given by

$$
N-\lim _{n \rightarrow \infty} \frac{f\left(k^{n} x\right)}{k^{2 n}}=Q(x)
$$

is well defined for all $x \in X$. In addition, it follows from the conditions (30), (31) and $\left(\mathrm{N}_{4}\right)$ that

$$
\begin{aligned}
N\left(\frac{D_{k l} f\left(k^{n} x, k^{n} y\right)}{k^{2 n}}, t\right) & \geq \min \left\{N^{\prime}\left(\varphi\left(k^{n} x\right), \frac{k^{2 n q} t^{q}}{2^{q}}\right), N^{\prime}\left(\varphi\left(k^{n} y\right), \frac{k^{2 n q} t^{q}}{2^{q}}\right)\right\} \\
& =\min \left\{N^{\prime}\left(|s|^{n} \varphi(x), \frac{k^{2 n q} t^{q}}{2^{q}}\right), N^{\prime}\left(|s|^{n} \varphi(y), \frac{k^{2 n q} t^{q}}{2^{q}}\right)\right\} \\
& =\min \left\{N^{\prime}\left(\varphi(x),\left(\frac{k^{2 q}}{|s|}\right)^{n} \frac{t^{q}}{2^{q}}\right), N^{\prime}\left(\varphi(y),\left(\frac{k^{2 q}}{|s|}\right)^{n} \frac{t^{q}}{2^{q}}\right)\right\} \\
& \rightarrow 1 \quad \text { as } n \rightarrow \infty, t>0,
\end{aligned}
$$

for all $x \in X$. Therefore we obtain, by use of $\left(\mathrm{N}_{4}\right),(33)$ and (34),

$$
\begin{aligned}
N\left(D_{k l} Q(x, y), t\right) & \geq \min \left\{N\left(D_{k l} Q(x, y)-\frac{D_{k l} f\left(k^{n} x, k^{n} y\right)}{k^{2 n}}, \frac{t}{2}\right), N\left(\frac{D_{k l} f\left(k^{n} x, k^{n} y\right)}{k^{2 n}}, \frac{t}{2}\right)\right\} \\
& \left.=N\left(\frac{D_{k l} f\left(k^{n} x, k^{n} y\right)}{k^{2 n}}, \frac{t}{2}\right) \quad \text { (for sufficiently large } n\right) \\
& \geq \min \left\{N^{\prime}\left(\varphi(x),\left(\frac{k^{2 q}}{|s|}\right)^{n} \frac{t^{q}}{4^{q}}\right), N^{\prime}\left(\varphi(y),\left(\frac{k^{2 q}}{|s|}\right)^{n} \frac{t^{q}}{4^{q}}\right)\right\} \\
& \rightarrow 1 \quad \text { as } n \rightarrow \infty, t>0,
\end{aligned}
$$

which implies $D_{k l} Q(x, y)=0$ by $\left(\mathrm{N}_{2}\right)$, and so the mapping $Q$ is quadratic satisfying equation (2).

(iii) The mapping $Q$ is a unique fixed point of the operator $J$ in the set $\Delta=\{g \in$ $\left.\Omega \mid d_{\Omega}(f, g)<\infty\right\}$. Thus if we assume that there exists another Euler-Lagrange type quadratic mapping $Q^{\prime}: X \rightarrow Y$ satisfying inequality (32), then

$$
Q^{\prime}(x)=\frac{Q^{\prime}(k x)}{k^{2}}=J Q^{\prime}(x), \quad d_{\Omega}\left(f, Q^{\prime}\right) \leq \frac{1}{\left(k^{2}-|s|^{\frac{1}{q}}\right)}<\infty,
$$


and so $Q^{\prime}$ is a fixed point of the operator $J$ and $Q^{\prime} \in \Delta=\left\{g \in \Omega \mid d_{\Omega}(f, g)<\infty\right\}$. By the uniqueness of the fixed point of $J$ in $\Delta$, we find that $Q=Q^{\prime}$, which proves the uniqueness of $Q$ satisfying inequality (32). This ends the proof of the theorem.

We observe that if $0<|s|<1$ in Theorem 4.1, then $\min \left\{N^{\prime}\left(\varphi(x), t^{q}\right), N^{\prime}\left(\varphi(0), t^{q}\right)\right\}=$ $N^{\prime}\left(\varphi(x), t^{q}\right)$ for all $x \in X$ and all $t>0$ since $N^{\prime}\left(\varphi(x), t^{q}\right) \geq N^{\prime}\left(\varphi(0), \frac{t^{q}}{|s|^{n}}\right) \rightarrow 1$ as $n \rightarrow \infty$ by (31).

Theorem 4.2 Assume that there exists a constant $s \in \mathbf{R}$ with $|s| \neq 1$ and $q>0$ satisfying $|s|^{\frac{1}{q}}>k^{2}$ such that a mapping $f: X \rightarrow Y$ with $f(0)=0$ satisfies the inequality

$$
N\left(D_{k l} f(x, y), t_{1}+t_{2}\right) \geq \min \left\{N^{\prime}\left(\varphi(x), t_{1}^{q}\right), N^{\prime}\left(\varphi(y), t_{2}^{q}\right)\right\}
$$

for all $x, y \in X, t_{i}>0(i=1,2)$ and that $\varphi: X \rightarrow Z$ is a mapping satisfying

$$
N^{\prime}\left(\varphi\left(\frac{x}{k}\right), t\right) \geq N^{\prime}\left(\frac{1}{s} \varphi(x), t\right)
$$

for all $x \in X$. Then there exists a unique quadratic mapping $Q: X \rightarrow Y$ satisfying the equation $D_{k l} Q(x, y)=0$ and the inequality

$$
N(f(x)-Q(x), t) \geq \min \left\{N^{\prime}\left(\frac{\varphi(x)}{\left(|s|^{\frac{1}{q}}-k^{2}\right)^{q}}, t^{q}\right), N^{\prime}\left(\frac{\varphi(0)}{\left(|s|^{\frac{1}{q}}-k^{2}\right)^{q}}, t^{q}\right)\right\}, \quad t>0,
$$

for all $x \in X$.

Proof The proof of this theorem is similar to that of Theorem 4.1.

\section{Competing interests}

The authors declare that they have no competing interests.

\section{Authors' contributions}

All authors carried out the proof. All authors conceived of the study, and participated in its design and coordination. All authors read and approved the final manuscript.

\section{Author details}

'Department of Mathematics, Chungnam National University, Daejeon, 305-764, Korea. ${ }^{2}$ Pedagogical Department E.E., National and Capodistrian University of Athens, 4 Agamemnonos Street, Aghia Paraskevi, Athens, 15342, Greece.

\section{Acknowledgements}

The authors would like to thank the referees and the editors for carefully reading this article and for their valuable comments. This research was supported by the Basic Research Program through the National Research Foundation of Korea (NRF) funded by the Ministry of Education (No. 2012R1A1A2008139).

Received: 26 October 2012 Accepted: 9 July 2013 Published: 1 August 2013

\section{References}

1. Bag, T, Samanta, SK: Finite dimensional fuzzy normed linear spaces. J. Fuzzy Math. 11(3), 687-705 (2003)

2. Felbin, C: Finite dimensional fuzzy normed linear spaces. Fuzzy Sets Syst. 48(2), 239-248 (1992)

3. Krishna, SV, Sarma, KKM: Separation of fuzzy normed linear spaces. Fuzzy Sets Syst. 63(2), 207-217 (1994)

4. Mirmostafaee, AK, Mirzavaziri, M, Moslehian, MS: Fuzzy stability of the Jensen functional equation. Fuzzy Sets Syst. $159,730-738(2008)$

5. Xiao, JZ, Zhu, XH: Fuzzy normed spaces of operators and its completeness. Fuzzy Sets Syst. 133(3), $389-399$ (2003)

6. Cheng, SC, Mordeson, JM: Fuzzy linear operators and fuzzy normed linear spaces. Bull. Calcutta Math. Soc. 86(5), 429-436 (1994)

7. Kramosil, I, Michalek, J: Fuzzy metric and statistical metric spaces. Kybernetika 11(5), 336-344 (1975) 
8. Bag, T, Samanta, SK: Fuzzy bounded linear operators. Fuzzy Sets Syst. 151, 513-547 (2005)

9. Mirmostafaee, AK, Moslehian, MS: Fuzzy versions of Hyers-Ulam-Rassias theorem. Fuzzy Sets Syst. 159, 720-729 (2008)

10. Mirzavaziri, M, Moslehian, MS: A fixed point approach to stability of a quadratic equation. Bull. Braz. Math. Soc. 37(3), 361-376 (2006)

11. Margolis, B, Diaz, JB: A fixed point theorem of the alternative for contractions on a generalized complete metric space. Bull. Am. Math. Soc. 126, 305-309 (1968)

12. Cădariu, L, Radu, V: Fixed points and the stability of Jensen's functional equation. J. Inequal. Pure Appl. Math. 4(1), Article ID 4 (2003)

13. Isac, G, Rassias, TM: Stability of $\psi$-additive mappings. Applications to nonlinear analysis. Int. J. Math. Math. Sci. 19(2), 219-228 (1996)

14. Park, C: Fixed points and Hyers-Ulam-Rassias stability of Cauchy-Jensen functional equations in Banach algebras. Fixed Point Theory Appl. 2007, Article ID 50175 (2007)

15. Radu, V: The fixed point alternative and the stability of functional equations. Fixed Point Theory Appl. 4(1), 91-96 (2003)

16. Xu, TZ, Rassias, JM, Rassias, MJ, Xu, WX: A fixed point approach to the stability of quintic and sextic functional equations in quasi-normed spaces. J. Inequal. Appl. 2010, Article ID 423231 (2010)

17. Pourpasha, MM, Rassias, JM, Saadati, R, Vaezpour, SM: A fixed point approach to the stability of Pexider quadratic functional equation with involution. J. Inequal. Appl. 2010, Article ID 839639 (2010)

18. Ulam, SM: A Collection of Mathematical Problems. Interscience, New York (1960)

19. Hyers, DH: On the stability of the linear functional equation. Proc. Natl. Acad. Sci. USA 27, 222-224 (1941)

20. Aoki, T: On the stability of the linear transformation in Banach spaces. J. Math. Soc. Jpn. 2, $64-66$ (1950)

21. Rassias, TM: On the stability of the linear mapping in Banach spaces. Proc. Am. Math. Soc. 72, 297-300 (1978)

22. Găvruta, P: A generalization of the Hyers-Ulam-Rassias stability of approximately additive mappings. J. Math. Anal. Appl. 184, 431-436 (1994)

23. Skof, F: Local properties and approximations of operators. Rend. Semin. Mat. Fis. Milano 53, 113-129 (1983)

24. Cholewa, PW: Remark on the stability of functional equations. Aequ. Math. 27, 76-86 (1984)

25. Czerwik, S: On the stability of the quadratic mapping in normed spaces. Abh. Math. Semin. Univ. Hamb. 62, 59-64 (1992)

26. Rassias, JM: On the stability of the non-linear Euler-Lagrange functional equation in real normed linear spaces. J. Math. Phys. Sci. 28, 231-235 (1994)

27. Rassias, JM: On the stability of the general Euler-Lagrange functional equation. Demonstr. Math. 29, 755-766 (1996)

28. Park, C, Cui, J: Generalized stability of $C^{*}$-ternary quadratic mappings. Abstr. Appl. Anal. 2007, Article ID 23282 (2007)

29. Eshaghi Gordji, M, Khodaei, H: On the generalized Hyers-Ulam-Rassias stability of quadratic functional equations. Abstr. Appl. Anal. 2009, Article ID 923476 (2009)

30. Park, C, Kim, J: The stability of a quadratic functional equation with the fixed point alternative. Abstr. Appl. Anal. 2009 Article ID 907167 (2009)

31. Lee, J, An, J, Park, C: On the stability of quadratic functional equations. Abstr. Appl. Anal. 2008, Article ID 628178 (2008)

32. Park, C: Generalized Hyers-Ulam stability of quadratic functional equations: a fixed point approach. Fixed Point Theory Appl. 2008, Article ID 493751 (2008)

33. Kim, H, Lee, J, Son, E: Approximate Euler-Lagrange quadratic mappings. J. Inequal. Appl. 2012, Article ID 58 (2012)

34. Eshaghi Gordji, M, Savadkouhi, M, Park, C: Quadratic-quartic functional equations in RN-spaces. J. Inequal. Appl. 2009, Article ID 868423 (2009)

35. Eshaghi Gordji, M, Abbaszadeh, S, Park, C: On the stability of a generalized quadratic and quartic type functional equation in quasi-Banach spaces. J. Inequal. Appl. 2009, Article ID 153084 (2009)

36. Kim, $\mathrm{H}$ : On the stability problem for a mixed type of quartic and quadratic functional equation. J. Math. Anal. Appl. 324, 358-372 (2006)

37. Hadžić, $\mathrm{O}$, Pap, E, Radu, V: Generalized contraction mapping principles in probabilistic metric spaces. Acta Math. Hung. 101(1-2), 131-148 (2003)

38. Mihet, D, Radu, V: On the stability of the additive Cauchy functional equation in random normed spaces. J. Math. Anal. Appl. 343, 567-572 (2008)

doi:10.1186/1029-242X-2013-358

Cite this article as: Kim et al.: Fuzzy approximation of Euler-Lagrange quadratic mappings. Journal of Inequalities and Applications 2013 2013:358. 